\title{
Arcs in cyclic affine planes
}

\author{
Vincenzo Giordano
}

go back

full screen

\section{Abstract}

In a cyclic affine plane of order $n$ with $n \equiv 1(\bmod 4)$, we construct a new family of $k$-arcs of size $k=\frac{1}{2}(n+7)$ containing $\frac{1}{2}(n+3)$ points from an oval.

Keywords: difference set, cyclic affine planes, arcs, ovals

MSC 2000: 05B10, 51E21, 51E15

\section{Introduction}

In a projective plane $\Pi$ of order $n$, an arc is defined to be a set of points no three of which are collinear. If the arc consists of $k \geq 3$ points, it is called a $k$-arc. An arc not contained in a larger one is said to be complete. The $(n+1)$-arcs are called ovals.

Arcs in the projective plane $\mathrm{PG}(2, q)$ coordinatized by a finite field $\mathrm{GF}(q)$ of order $q$ and their generalizations in higher dimensional projective spaces $\mathrm{PG}(n, q)$ are also relevant in Coding Theory, arcs and maximum distance separable codes being equivalent objects.

By a well known result due to B. Segre, $\frac{1}{2}(n+3)$ is the maximum number of points that an arc in a projective plane $\Pi$ of odd order $n$ can share with an oval $\mathcal{C}$ of $\Pi$. If the maximum is attained by a $k$-arc $\mathcal{K}$, then $\mathcal{K}$ seems to contain only a few points outside $\mathcal{C}$. In $\mathrm{PG}(2, q)$, this emerged from previous work by B. Segre, L. Lombardo-Radice, G. Pellegrino, G. Korchmáros and A. Sonnino; see $[4,5,6,7,9]$.

It is also known, that if $k=\frac{1}{2}(n+5)$ and $|\mathcal{K} \cap \mathcal{C}|=\frac{1}{2}(n+3)$, then $\mathcal{K}$ is complete in almost all cases, that is, the probability of finding such a $k$-arc which is not complete is equal to zero. This suggests that the problem of constructing $k$-arcs 




Theorem 4.1. Let $n \equiv 1(\bmod 4)$. In $\Pi$, the point-set

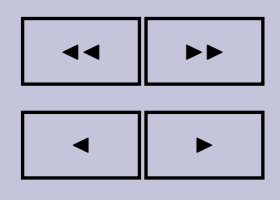

page $6 / 7$

go back

full screen

close

quit

$$
\begin{aligned}
\mathcal{K}:= & \left\{\overline{0}, \overline{2(n-1)}, \ldots, \overline{\frac{(n-5)(n-1)}{2}}, \frac{\overline{(n-1)(n-1)}}{2}, \frac{\overline{(n+1)(n-1)}}{2}\right. \\
& \frac{(n+5)(n-1)}{2}, \ldots, \overline{(n-2)(n-1)}, \overline{n(n-1)}, \rho\left(\overline{\left.\frac{n+1}{2}\right)}, \rho\left(\overline{\frac{n+5}{2}}\right)\right\}
\end{aligned}
$$

is an arc of size $\frac{1}{2}(n+7)$ containing $\frac{1}{2}(n+3)$ points of the oval $\Omega$.

Proof. For each $j=1, \ldots, \frac{n-1}{2}$ let $S_{j}$ be the line of $\Pi$ through the points $\overline{j(n-1)}$ and $\overline{(n-j+1)(n-1)}$. From the congruence

$$
j n(n-1) \equiv(n-j+1)(n-1) \quad(\bmod v)
$$

the collineation $\phi_{n}$ fixes the line $S_{j}$. Since $\phi_{n}$ is an involutory $\left(\rho\left(\frac{\overline{n+1}}{2}\right), N\right)$-homology, see Theorem 3.1, the lines fixed by $\phi_{n}$ are the axis $N$ and all the lines through the center $\rho\left(\frac{n+1}{2}\right)$. Therefore, the $\frac{n-1}{2}$ lines through $\rho\left(\frac{\overline{n+1}}{2}\right)$ and the chords of $\Omega$ are just the above defined lines $S_{j}$.

For each $k=0, \ldots, \frac{n-3}{2}$, let $R_{k}$ be the line of $\Pi$ through $\overline{k(n-1)}$ and $\overline{(n-k-1)(n-1)}$. From the congruence

$$
k n(n-1)+(n-1)(n-1) \equiv(n-k-1)(n-1) \quad(\bmod v)
$$

the collineation $\varepsilon_{n-1}$ fixes the line $R_{k}$. Since $\varepsilon_{n-1}$ is an involutory $\left(\rho\left(\overline{\frac{n+5}{2}}\right), N-\right.$ $\overline{n-1})$-homology, see Theorem 3.1, the $\frac{n-1}{2}$ lines through $\rho\left(\frac{n+5}{2}\right)$ which are 2-secant of $\Omega$ are just the above defined lines $R_{k}$. Finally, the lines through $\rho\left(\frac{\overline{n+1}}{2}\right)$ and $\rho\left(\frac{\overline{n+5}}{2}\right)$ which are 2 -secant of $\Omega$ meet $\Omega$ in two points $a$ and $b$ such that

- $a$ and $b$ are both even or odd multiples of $\overline{n-1}$,

- $a \in \mathcal{K} \cap \Omega$ and $b \in \overline{\mathcal{K}} \cap \Omega$.

This completes the proof.

We remark that the translation $f_{(n+1) / 2}$ mapping $\bar{x}$ to $\overline{x+v / 2}$, is an involutory $\left(L_{\infty}, \infty\right)$-homology preserving the oval $\Omega$ and leaving $\mathcal{K}$ invariant.

Remark 4.2. It has been conjectured that every cyclic affine plane is desarguesian, and this has been proven for affine cyclic planes of order $n \leq 1000$. If $\pi$ is the projective closure $\mathrm{PG}(2, n)$ of the affine plane $\mathrm{AG}(2, n)$ with $n \equiv 1(\bmod 4)$, by the famous Segre's theorem, the oval $\Omega$ defined in Theorem 3.1 is a conic. 
